\title{
Automated Segmentation of Hippocampal Subfields in Drug-Naïve Patients with Alzheimer Disease
}

\author{
H.K. Lim, S.C. Hong, W.S. Jung, K.J. Ahn, W.Y. Won, C. Hahn, I.S. Kim, and C.U. Lee
}

\begin{abstract}
BACKGROUND AND PURPOSE: Although a few automated hippocampal subfield segmentation methods have been developed, there is no study on the effects of the diagnosis of Alzheimer disease on the hippocampal subfield volume with in vivo MR imaging. The aim of this study was to investigate hippocampal subfield volume differences between drug-naive subjects with $A D$ and healthy elderly controls by using an automated hippocampal subfield segmentation technique.
\end{abstract}

MATERIALS AND METHODS: Thirty-one drug-naïve subjects with AD and 33 group-matched healthy control subjects underwent $3 T$ MR imaging, and hippocampal subfield volume was measured and compared between the groups.

RESULTS: Subjects with AD had significantly smaller volumes of the presubiculum, subiculum, CA2-3, and CA4 DG compared with healthy subjects (uncorrected, $P<.001$ ). In addition, we found significant positive correlations between the presubiculum and the subicular volumes and the MMSE-K and the CERAD-K verbal delayed recall scores in the AD group.

CONCLUSIONS: We are unaware of previous imaging studies of automated hippocampal subfield segmentation in AD. These structural changes in the hippocampal presubiculum, subiculum, and CA2-3 might be at the core of underlying neurobiologic mechanisms of hippocampal dysfunction and their relevance to verbal delayed recall impairments in AD.

ABBREVIATIONS: $\mathrm{AD}=$ Alzheimer disease; $\mathrm{aMCI}=$ amnestic mild cognitive impairment; $\mathrm{CA}=$ cornu ammonis; $\mathrm{CERAD}-\mathrm{K}=$ Korean version of the Consortium to Establish a Registry for Alzheimer's Disease; DG = dentate gyrus; MMSE-K = Korean version of Mini-Mental State Examination

A lzheimer disease is the most common cause of dementia in the elderly. The hippocampus, part of the medial temporal lobe memory system, is particularly vulnerable to damage at the very earliest stages of $\mathrm{AD} .{ }^{1,2}$ Hippocampal atrophy is, therefore, considered an important biomarker of $\mathrm{AD}$. In addition, previous pathologic studies showed that the atrophic pattern in the hippocampus in $\mathrm{AD}$ was rather selective; that is, degeneration within the CA1 and subiculum regions appeared to be more severe compared with that in other components of the hippocampus in the early stages of $\mathrm{AD} .{ }^{3}$ In addition, several previous studies have tried to identify hippocampal atrophy of $\mathrm{AD}$ in vivo by measuring total

Received May 2, 2012; accepted after revision July 2

From the Departments of Psychiatry (H.K.L., S.C.H.) and Radiology (W.S.J.), Saint Vincent Hospital, Suwon, College of Medicine, Catholic University of Korea, Suwon, Korea; Departments of Radiology (K.J.A.) and Psychiatry (C.H., C.U.L.), Seoul Saint Mary's Hospital, Seoul, College of Medicine, Catholic University of Korea, Seoul, Korea; Department of Psychiatry (W.Y.W.), Saint Paul's Hospital, Seoul, College of Medicine, Catholic University of Korea, Seoul, Korea; and Siemens Healthcare (I.S.K.), Seoul, Korea.

Please address correspondence to Chang Uk Lee. MD, PhD, Department of Psychiatry, Seoul Saint Mary's Hospital, Seoul, College of Medicine, Catholic University of Korea 505 Banpo dong Seochogu, Seoul, 137-701 Republic of Korea; e-mail: jihan@catholic.ac.kr

http://dx.doi.org/10.3174/ajnr.A3293 volumes and 3D surface mapping, and their results were consistent with those in the postmortem studies. However, because the morphology of the hippocampus is convoluted, volumetric measurement of the inner subfields was needed to understand the complex anatomic change of the hippocampus. ${ }^{4}$ Using manual delineation of hippocampal subfields, Mueller and Weiner ${ }^{5}$ reported that subfield atrophy occurred in the CA1-2 boundary zone in amnestic mild cognitive impairment and in CA1 in AD. Although manual subfield segmentation provides the most accurate delineation of hippocampal subfields, it requires labor-intensive work and is, therefore, not suitable for dealing with a large amount of MR imaging data. ${ }^{4,6}$

To overcome these problems, a few researchers have developed automated hippocampal subfield segmentation methods. ${ }^{4,7}$ The method of Van Leemput et $\mathrm{al}^{7}$ was based on Markov random fields prior to labeling the hippocampal subfields and was validated by comparison with manual tracing. In this study, an atlas mesh was previously built from the manual delineation of the hippocampus in 10 control subjects. The Dice overlap measures between manual and automated segmentation methods were approximately 0.7 for all substructures (from CA2-3 and the subiculum at 0.74 to CA1 at 0.62$).{ }^{7}$ In another preliminary study on 
subjects with aMCI by using the same method, Hanseeuw et $\mathrm{al}^{6}$ showed that CA2-3 and subicular volumes were significantly smaller in subjects with aMCI, while other subfields were not, compared with healthy controls.

We are unaware of previous imaging studies of automated hippocampal subfield segmentation in $\mathrm{AD}$. The aim of this study was to compare hippocampal subfield volume differences between controls and drug-naïve subjects with $\mathrm{AD}$ with a somewhat larger sample size by using the automated hippocampal subfield segmentation method of Van Leemput et al. ${ }^{7}$ Indeed, a previous study revealed that the hippocampal atrophy rate might be affected by medications such as cholinesterase inhibitors. ${ }^{8}$ We hypothesized that CA1 and subiculuar volumes would be smaller while other subfields would be spared in $\mathrm{AD}$ compared with controls.

\section{MATERIALS AND METHODS Subjects}

In this study, 31 patients with $\mathrm{AD}$ were included. They were recruited through the outpatient psychogeriatric clinic of St. Vincent Hospital located in Suwon, South Korea, from October 2009 to October 2010. The patient group fulfilled the National Institute of Neurologic and Communication Disorders and Stroke/Alzheimer Disease and Related Disorders Association criteria for probable $\mathrm{AD}^{9}$ and had a score on the Clinical Dementia Rating Scale of $\geq 1$. ${ }^{10}$ We excluded from the study subjects who had other neurologic or psychiatric conditions (including other forms of dementia or depression) and those taking any psychotropic medications (eg, cholinesterase inhibitors, antidepressants, benzodiazepines, and antipsychotics). Subjects were screened with a selfreport health questionnaire that reviewed both the demographic data and the medical history.

Thirty-three healthy controls were recruited within the community through an advertisement in the local newspaper. Control subjects were matched to patients on age, handedness, and level of education. Furthermore, control subjects were given the same self-report health questionnaire as the patients, thus enabling matching on health status.

The study was conducted in accordance with the ethical and safety guidelines set forth by the local institutional review board of Catholic University of Korea. Informed consent was obtained from all subjects and their guardians participating in the study. All subjects were right-handed.

\section{MR Imaging Acquisition}

All participants underwent MR imaging on a 3T whole-body scanner equipped with a 32-channel head coil (Verio; Siemens, Erlangen, Germany). The scanning parameters of the T1weighted optimized high-resolution 3D magnetization-prepared rapid acquisition of gradient echo sequences were as follows: TR/
$\mathrm{TI} / \mathrm{TE}=1900 / 900 / 2.5 \mathrm{~ms}, \mathrm{FOV}=250 \times 250 \mathrm{~mm}$, flip angle $=9^{\circ}$, 208 sections acquired coronally, thickness $=0.8 \mathrm{~mm}$, acquisition time $=7.34$ minutes.

\section{Image Processing}

For cortical reconstruction and volumetric segmentation of the whole brain, the FreeSurfer image-analysis suite (Version 5.0.1; http://surfer.nmr.mgh.harvard.edu), documented and freely available on-line, was used. The technical details of these procedures have been described in previous publications. ${ }^{11,12}$ Briefly, the processing stream includes a Talairach transform of each subject's native brain, removal of the nonbrain tissue, and segmentation of the gray matter-white matter tissue. The cortical surface of each hemisphere was inflated to an average spheric surface to locate both the pial surface and the gray matter-white matter boundary. We visually inspected the entire cortex of each subject and manually corrected any topologic defects, blinded to the subject's identity.

Hippocampal volumes were obtained from the automated procedure for volumetric measures of the brain structures implemented in FreeSurfer. Automated segmentation of the hippocampus to its respective subfields was performed by using Bayesian inference and a statistical model of the medial temporal lobe. The left and right hippocampi were segmented into 7 subfields: CA1, CA2-3, CA4-DG, subiculum, presubiculum, fimbria, and hippocampal fissure (Fig 1).

\section{Statistical Analyses}

Statistical analyses for demographic data were performed with the Statistical Package for the Social Sciences software (Version 12.0; SPSS, Chicago, Illinois). Assumptions for normality were tested for all continuous variables. Normality was tested by using the 
Table 1: Demographic and clinical characteristics of study participants

\begin{tabular}{lccc}
\hline & Control Group $(\boldsymbol{n}=\mathbf{3 3})$ & AD Group $(\boldsymbol{n}=31)$ & $\boldsymbol{P}$ Value \\
\hline Age $(\mathrm{yr} \pm \mathrm{SD})$ & $75.6 \pm 4.2$ & $75.0 \pm 8.5$ & $\mathrm{NS}$ \\
Education $(\mathrm{yr} \pm \mathrm{SD})$ & $9.1 \pm 4.7$ & $9.7 \pm 4.17$ & $\mathrm{NS}$ \\
Sex $(\mathrm{M} / \mathrm{F})$ & $14: 19$ & $13: 18$ & $\mathrm{NS}$ \\
CDR & 0 & $1.6 \pm 0.7$ & \\
MMSE-K & $29.3 \pm 0.3$ & $23.4 \pm 3.2$ & $<.0001$ \\
CERAD-K verbal delayed recall & $6.9 \pm 1.8$ & $2.9 \pm 1.6$ & $<.0001$ \\
TICV $\left(\mathrm{mm}^{3}\right)$ & $1388069.9 \pm 131940.8$ & $1319932.8 \pm 124204.3$ & 0.038 \\
\hline
\end{tabular}

Note:-NS indicates not significant; CDR, Clinical Dementia Rating; TICV, total intracranial volume sent, was approximately the equivalent to $P<.05$, corrected for multiple comparisons. ${ }^{16,17}$

\section{RESULTS}

\section{Demographic Data}

Table 1 shows the baseline demographic data in our different subject groups. There was no significant difference in sex, age, and education between the $\mathrm{AD}$ group and the control
Table 2: Normalized hippocampal subfield volume of control and AD groups

\begin{tabular}{|c|c|c|c|c|}
\hline \multirow[b]{2}{*}{ Subfields } & \multicolumn{2}{|c|}{ Volume $\left(\mathrm{mm}^{3}, \mathrm{SD}\right)$} & \multirow[b]{2}{*}{$\begin{array}{c}\% \\
\text { Difference }\end{array}$} & \multirow[b]{2}{*}{$\begin{array}{c}P \\
\text { Value }\end{array}$} \\
\hline & $\begin{array}{c}\text { Control Group } \\
(n=33)\end{array}$ & $\begin{array}{c}\text { AD Group } \\
(n=31)\end{array}$ & & \\
\hline \multicolumn{5}{|l|}{ Left } \\
\hline Total $^{\mathrm{a}}$ & 3818.7 (441.3) & $3068.6(525.0)$ & 19.6 & $<.0001^{\mathrm{a}}$ \\
\hline Presubiculum $^{\mathrm{a}}$ & $425.4(50.8)$ & $323.2(69.0)$ & 24.0 & $.001^{\mathrm{a}}$ \\
\hline Subiculum ${ }^{a}$ & $609.7(72.6)$ & $501.4(90.2)$ & 17.7 & $<.0001^{\mathrm{a}}$ \\
\hline CAl & 322.4 & $311.5(4 \varepsilon$ & 3.3 & .347 \\
\hline $\mathrm{CA} 2-3$ & $902.4(112$ & 785.0 (118.6) & 13.0 & .009 \\
\hline CA4-DG & $509.7(64.2)$ & $440.3(65.3)$ & 13.6 & .006 \\
\hline Fimbria & 58.8 (18.8) & $48.4(2$ & 17.4 & .145 \\
\hline Fissure & $37.3(15$ & 40.1 & 7.7 & .716 \\
\hline \multicolumn{5}{|l|}{ Right } \\
\hline Total $^{\mathrm{a}}$ & 3984.8 & 3077.6 & 22.7 & $<.0001^{\mathrm{a}}$ \\
\hline Presubiculum $^{a}$ & $416.1(48.3)$ & 319.0 & 23.3 & $<.0001^{\mathrm{a}}$ \\
\hline Subiculum $^{a}$ & $629.3(69.8)$ & 502.5 (107.5) & 20.1 & $<.0001^{\mathrm{a}}$ \\
\hline CAl & $334.7(49.2)$ & $311.1(65.3)$ & 7.0 & .022 \\
\hline $\mathrm{CA} 2-3^{\mathrm{a}}$ & 973.8 (131.6) & 814.3 (164.4) & 16.3 & $<.0001^{a}$ \\
\hline CA4-DG ${ }^{\mathrm{a}}$ & 544.9 (71.5) & $457.9(87.3)$ & $15.9 \%$ & $<.0001^{\mathrm{a}}$ \\
\hline Fimbria & $47.2(15.3)$ & $41.6(20.3)$ & $11.8 \%$ & .059 \\
\hline Fissure & 45.5 (17.7) & $47.8(22.4)$ & $5.0 \%$ & .720 \\
\hline
\end{tabular}

${ }^{a}$ Uncorrected $P<.001$ was considered significant.

Kolmogorov-Smirnov test. All variables were normally distributed. The independent $t$ test and the $\chi^{2}$ test were used to assess potential differences between the $\mathrm{AD}$ and healthy control groups for all demographic variables. All statistical analyses had a 2-tailed $\alpha$ level of $<.05$ for defining statistical significance. In accordance with other volumetric analyses, adjustment was performed for each region by an analysis of covariance approach: adjusted volume $=$ raw volume $-\mathrm{b} \times($ intracranial volume $[\mathrm{ICV}]-$ mean ICV), where $b$ is the slope of a regression of a region-of-interest volume on ICV. ${ }^{13}$ Adjusted volume was used for all analyses described in this study.

To assess both the main effects of diagnosis (AD-versus-control) factors on the hippocampal subfield volume, we used analysis of covariance with total intracranial volume, education, sex, and age as nuisance variables. In addition, regression analyses were performed to determine the contribution of clinical variables (Korean version of the Mini-Mental State Examination ${ }^{14}$ and the Korean version of the Consortium to Establish a Registry for Alzheimer disease $)^{15}$ delayed recall scores) to hippocampal subfield volume. Because the FreeSurfer analysis suite does not provide correction for multiple comparisons in the analysis of hippocampal subfield volume, we set an uncorrected $P<.001$ (2-tailed) as a significant threshold in the statistical difference maps. This threshold, when an a priori hypothesis was pre- group. Compared with the control group, the $\mathrm{AD}$ group showed significantly poorer performances on the MMSE-K and the CERAD-K delayed recall test $(P<.0001)$.

\section{Quantitative Volumetric Data}

The total volumes of the left $(F=19.3 ; P<.0001)$ and the right $(F=32.2 ; P<.0001)$ hippocampi of the AD group were significantly smaller than those of the control group (Table 2).

In the left hippocampus, the presubiculum $(F=13.2 ; P=$ $.001)$ and the subiculum $(F=18.2 ; P<.0001)$ volumes were significantly smaller in the $\mathrm{AD}$ group compared with the control group. In addition, the $\mathrm{AD}$ group had a smaller volume tendency in the left CA2-3 $(F=7.1 ; P=.009)$ and the CA4-DG $(F=8.2$; $P=.006)$ compared with the controls. However, there were no significant volume differences between the $\mathrm{AD}$ group and control group in the left CA1 $(F=0.8 ; P=.347)$, the fimbria $(F=2.1 ; P=$ $.145)$, and the hippocampal fissure $(F=0.1 ; P=.716)$.

In the right hippocampus, the presubiculum $(F=29.5 ; P<$ $.0001)$, the subiculum $(F=24.9 ; P<.0001)$, the CA2-3 $(F=15.8$; $P<.0001)$, and the CA4-DG volumes $(F=15.5 ; P<.0001)$ were significantly smaller in the $\mathrm{AD}$ group compared with the control group. In addition, the $\mathrm{AD}$ group had a smaller volume tendency in the right CA1 $(F=5.5 ; P=.022)$ compared with the controls. However, there were no significant volume differences between the $\mathrm{AD}$ group and the control group in the right fimbria $(F=3.7$; $P=.059)$ and the hippocampal fissure $(F=0.1 ; P=.720)$.

In the correlation analysis, age was not correlated significantly with any hippocampal subfield volume in the AD group. However, the left total hippocampal $(r=-0.46, P=.001)$ and the CA4-DG $(r=-0.48 ; P=.001)$ volumes were negatively correlated significantly with age in the control group. The MMSE-K scores of the subjects with AD were positively correlated significantly with the left subiculum $(r=0.47 ; P=.001)$ and the total hippocampal volume $(r=0.48 ; P=.001)$; and the right subicu$\operatorname{lum}(r=0.84 ; P<.0001)$, the presubiculum $(r=0.43 ; P=.001)$, the CA2-3 $(r=0.43 ; P=.001)$, and the total hippocampal volume $(r=0.55 ; P<.0001)$. In addition, the CERAD-K delayed recall scores of the subjects with $\mathrm{AD}$ were positively correlated with the left presubiculum $(r=0.53 ; P<.0001)$, subiculum $(r=$ $0.59 ; P<.0001)$, CA4-DG $(r=0.45 ; P=.001)$, and total hippocampal volume $(r=0.49 ; P=.001)$

No significant correlation was observed between the other hippocampal subfield volumes and the MMSE-K scores and the CERAD-K neuropsychological test scores in the $\mathrm{AD}$ group. In addition, no significant correlations were observed between the CERAD-K neuropsychological test scores and the hippocampal subfield volume in the control group.

AJNR Am J Neuroradiol 34:747-51 Apr 2013 www.ajnr.org 


\section{DISCUSSION}

We are unaware of previous imaging studies on the effect of diagnosis of AD on the hippocampal subfields by using the automated hippocampal subfield segmentation method by Van Leemput et al. ${ }^{7}$ In this study, the subjects with AD had significantly smaller volumes of the presubiculum, the subiculum, the CA2-3, and the CA4-DG compared with the healthy subjects.

To date, there was a study on the hippocampal subfield volume segmentation analysis in $\mathrm{AD} .{ }^{5}$ Using manual segmentation analysis of hippocampal subfield volume, Mueller and Weiner ${ }^{5}$ reported that patients with $\mathrm{AD}$ had smaller subicular and CA1 volumes, compared with controls, which is in line with those in our study. In the progression from mid cognitive impairment to $\mathrm{AD}$, neurodegeneration is known to occur in a progressive and hierarchic fashion. ${ }^{18}$ Among the hippocampal subfields, degeneration within the CA1 and subiculum appeared to be more severe compared with other components of the hippocampus in aMCI and the early stages of $\mathrm{AD} .^{3}$ Pathologic findings in patients with $\mathrm{AD}$ suggested that severe degeneration of the perforant path providing input from layer III of the entorhinal cortex to the CA1 and the subiculum was a characteristic feature of AD. ${ }^{18,19}$

The presubiculum, which can be defined as a modified 6-layered cortex between the subiculum and the main part of the parahippocampal gyrus, may play a role in processing episodic memory. ${ }^{20}$ In addition, the previous neuropathologic studies showed that regions including the entorhinal transition area and the presubiculum contain exceptionally high levels of amyloid plaques in patients with $\mathrm{AD} .{ }^{21}$ A previous study by Hanseeuw et $\mathrm{al}^{6}$ showed smaller right presubicular volume in patients with aMCI by using the same methods as ours, compared with controls. Therefore, measuring presubicular volume might be as sensitive as measuring subicular and CA1 volume in detecting pathologic processes of $\mathrm{AD}$ and aMCI. However, further longitudinal study and discrimination analysis with larger samples will be needed.

Contrary to our hypothesis, we could not observe any significant volume difference in the CA1 subfields between the aMCI group and controls, which agreed with previous studies on aMCI by Hanseeuw et al by using the same method. ${ }^{6}$ However, these results were not in line with those in previous studies using the manual segmentation of hippocampal subfields or 3D surface mapping of the hippocampus. ${ }^{5,22}$ As to this discrepancy, Hanseeuw et al suggested that there was a boundary difference between the studies and possible CA1 hypertrophy in aMCI. They also suggested that the reproducibility of their method would permit more consistency between studies than was possible with manual methods. Similar patterns of our results compared with those in the study by Hanseeuw et al might confirm the reproducibility of the automated hippocampal subfield segmentation method by Van Leemput et al. ${ }^{6,7}$ The method by Van Leemput et $\mathrm{al}^{7}$ used geometric rules as criteria for defining subfields (presubiculum, subiculum, CA1, CA2-3, and CA4-DG), whereas the protocol used by Mueller and Weiner ${ }^{5}$ generally proceeded by comparing in vivo image sections with the annotated postmortem data by Duvernoy et al. ${ }^{23}$ Furthermore, the anatomic definition of hippocampal subfield boundaries in $3 \mathrm{D}$ surface mapping also used the atlas of Duvernoy et al. ${ }^{23,24}$ Indeed, there were differences in defining the CA1 and subiculum in the previous studies; for example, what was defined as CA1 in the study by Mueller and Weiner included a substantial proportion of what was defined as the subiculum in the study by Van Leemput et al. In addition, what was defined as subiculum in the study by Mueller and Weiner seemed to include a substantial portion of the presubiculum as defined by Van Leemput et al.

According to the anatomic atlases, the CA1 is one of the largest hippocampal subfields. ${ }^{23}$ However, as indicated in Table 2, the CA1 volume was smaller than the CA2-3 volume. Previous postmortem studies reported that the $\mathrm{CA} 2-3$ regions are much smaller than the CA1 region. ${ }^{23,25}$ Although the segmentation protocol by Van Leemput et $\mathrm{al}^{7}$ was validated with manual segmentation, there might be the possibility of definition bias in the hippocampal subfield boundaries in their study. ${ }^{7}$ Because the previous postmortem pathologic studies indicated that degeneration within the CA1 and subiculum appeared to be more severe compared with other components of the hippocampus in aMCI and early stages of $\mathrm{AD}$, further consensus in defining the subfield boundary on in vivo MR imaging will be needed. ${ }^{18}$ In addition, the study by Hanseeuw et $\mathrm{al}^{6}$ reported that the hippocampal subfield segmentation of the subiculum, the presubiculum, and the CA2-3 might have some discriminative power in diagnosing aMCI. ${ }^{6}$ However, these results should be interpreted cautiously because there are mismatches in defining subfield boundaries between the segmentation protocol by Van Leemput et $\mathrm{al}^{7}$ and the anatomic atlases. ${ }^{23}$ In this regard, we could not determine the diagnostic value of the hippocampal subfield segmentation in AD. Because the previous study by Mueller and Weiner ${ }^{5}$ reported that the manual segmentation had substantial diagnostic power in detecting $\mathrm{AD}$ and aMCI, a more accurate anatomic boundary definition will increase the power of the diagnostic value of the method by Van Leemput et al. ${ }^{7,26}$

In this study, the $\mathrm{AD}$ group showed significant correlation between the presubiculum, subiculum, and total hippocampal volumes and CERAD-K verbal delayed recall scores. Although there was no study on the relationships between the hippocampal subfield volume and verbal delayed recall scores in $\mathrm{AD}$, the results were in line with those in the previous study using the 3D surface mapping in aMCI. ${ }^{22}$ An automated hippocampal shape-analysis method by using a pattern-recognition algorithm showed a positive correlation between CERAD delayed recall scores and hippocampal deformation in the CA1 and subiculum. ${ }^{22}$ In addition, the left total hippocampal and CA4-DG volumes were negatively correlated significantly with age in the control group, which was also in accordance with those in the previous studies. ${ }^{5,6}$ The study by Hanseeuw et $\mathrm{al}^{6}$ showed that total hippocampal volume was significantly explained by age in the healthy elderly controls. Additionally, they showed that age tended to explain CA4-DG volume in the controls. ${ }^{6}$ In the manual hippocampal subfield segmentation study by Mueller and Weiner, ${ }^{5}$ they reported that CA3-DG volumes remained stable up to the fifth decade and then became increasingly smaller.

Our study had some limitations. First, although we could quantitatively analyze the hippocampal subfield volume differences between the $\mathrm{AD}$ and controls, we could not see the anatomic locations of the morphologic changes. Therefore, further analysis of hippocampal subfield segmentation with 3D surface mapping 
will provide more accurate detection of hippocampal morphologic changes in AD. Second, because we set the statistical significance threshold as $P<.001$ for equivalence of the false discovery rate correction $P<.05$, we could not exclude the type II errors in the results. Indeed, we excluded the subfields with $P$ values between 0.05 and 0.001 , such as the left hippocampal CA2-3 and CA4-DG, and the right hippocampal CA1. Hence, larger samples will be needed to investigate subtle differences in the hippocampal subfields between patients with $\mathrm{AD}$ and controls. Third, because the FreeSurfer analysis suite does not provide a bias-correction process in the hippocampal subfield segmentation, we could not conduct image correction of our subjects. Although we could not find any serious errors in the automated hippocampal subfield segmentation after thorough inspection of the image quality of our subjects, there would be somewhat serious problems in the feasibility of the method by Van Leemput et $\mathrm{al}^{7}$ in patients with dementia with substantial hippocampal atrophy. Therefore, an automated or manual bias-correction process of hippocampal subfield segmentation in the FreeSurfer software will be needed for more precise labeling of the hippocampal subfields. Fourth, the validation study of the automated subfield segmentation with manual segmentation by Van Leemput et $\mathrm{al}^{7}$ was conducted by using ultra-high-resolution T1-weighted images acquired with a $>35$-minute acquisition time. Therefore, a validation study with manual segmentation in conventional-resolution MR imaging data with a short scanning time will be needed.

\section{REFERENCES}

1. Squire LR, Zola Morgan S. The medial temporal lobe memory system. Science 1991;253:1380-86

2. Braak H, Braak E. Neuropathological stageing of Alzheimer-related changes. Acta Neuropathol 1991;82:239-59

3. Li S, Shi F, Pu F, et al. Hippocampal shape analysis of Alzheimer disease based on machine learning methods. AJNR Am J Neuroradiol 2007;28:1339-45

4. Yushkevich PA, Wang H, Pluta J, et al. Nearly automatic segmentation of hippocampal subfields in in vivo focal T2-weighted MRI. Neuroimage 2010;53:1208-24

5. Mueller SG, Weiner MW. Selective effect of age, Apo e4, and Alzheimer's disease on hippocampal subfields. Hippocampus 2009;19: 558-64

6. Hanseeuw BJ, Van Leemput K, Kavec M, et al. Mild cognitive impairment: differential atrophy in the hippocampal subfields. AJNR Am J Neuroradiol 2011;32:1658-61

7. Van Leemput K, Bakkour A, Benner T, et al. Automated segmentation of hippocampal subfields from ultra-high resolution in vivo MRI. Hippocampus 2009;19:549-57

8. Wang L, Harms MP, Staggs JM, et al. Donepezil treatment and changes in hippocampal structure in very mild Alzheimer disease. Arch Neurol 2010;67:99-106

9. McKhann G, Drachman D, Folstein M, et al. Clinical diagnosis of
Alzheimer's disease: report of the NINCDS-ADRDA Work Group under the auspices of Department of Health and Human Services Task Force on Alzheimer's Disease. Neurology 1984;34:939-44

10. Morris JC. The Clinical Dementia Rating (CDR): current version and scoring rules. Neurology 1993;43:2412-14

11. Dale AM, Fischl B, Sereno MI. Cortical surface-based analysis. I. Segmentation and surface reconstruction. Neuroimage 1999;9: 179-94

12. Fischl B, Dale AM. Measuring the thickness of the human cerebral cortex from magnetic resonance images. Proc Natl Acad Sci U S A 2000;97:11050-55

13. Raz N, Lindenberger U, Rodrigue KM, et al. Regional brain changes in aging healthy adults: general trends, individual differences and modifiers. Cereb Cortex 2005; 15:1676-89

14. Park J, Kwon Y. Modification of the Mini-Mental State Examination for use in the elderly in a non-western society. Part I. Development of Korean version of Mini-Mental State Examination. Int J Geriatr Psychiatry 1990;5:381-87

15. Lee JH, Lee KU, Lee DY, et al. Development of the Korean version of the Consortium to Establish a Registry for Alzheimer's Disease Assessment Packet (CERAD-K): clinical and neuropsychological assessment batteries. J Gerontol B Psychol Sci Soc Sci 2002;57:P47-53

16. Lyoo IK, Sung YH, Dager SR, et al. Regional cerebral cortical thinning in bipolar disorder. Bipolar Disord 2006;8:65-74

17. Ashburner J, Csernansky JG, Davatzikos C, et al. Computer-assisted imaging to assess brain structure in healthy and diseased brains. Lancet Neurol 2003;2:79-88

18. Braak E, Braak H. Alzheimer's disease: transiently developing dendritic changes in pyramidal cells of sector CA1 of the Ammon's horn. Acta Neuropathol 1997;93:323-25

19. Mizutani T, Kasahara M. Hippocampal atrophy secondary to entorhinal cortical degeneration in Alzheimer-type dementia. Neurosci Lett 1997;222:119-22

20. Malkova L, Mishkin M. One-trial memory for object-place associations after separate lesions of hippocampus and posterior parahippocampal region in the monkey. J Neurosci 2003;23:1956-65

21. Wisniewski HM, Sadowski M, Jakubowska Sadowska K, et al. Diffuse, lake-like amyloid-beta deposits in the parvopyramidal layer of the presubiculum in Alzheimer disease. J Neuropathol Exp Neurol 1998; 57:674-83

22. Costafreda SG, Dinov ID, Tu Z, et al. Automated hippocampal shape analysis predicts the onset of dementia in mild cognitive impairment. Neuroimage 2011;56:212-19

23. Duvernoy HM, Vannson LL, Cattin F. The Human Hippocampus, Functional Anatomy, Vascularization and Serial Sections with MRI. Berlin, Germany: Springer-Verlag; 2005

24. Frisoni GB, Sabattoli F, Lee AD, et al. In vivo neuropathology of the hippocampal formation in AD: a radial mapping MR-based study. Neuroimage 2006;32:104-10

25. Simić G, Kostović I, Winblad B, et al. Volume and number of neurons of the human hippocampal formation in normal aging and Alzheimer's disease. J Comp Neurol 1997;379:482-94

26. Mueller SG, Schuff N, Yaffe K, et al. Hippocampal atrophy patterns in mild cognitive impairment and Alzheimer's disease. Human Brain Mapp 2010;31:1339-47 